\title{
Proposing WPOD-NET combining SVM system for detecting car number plate
}

\author{
Phat Nguyen Huu, Cuong Vu Quoc \\ School of Electronics and Telecommunications, Hanoi University of Science and Technology (HUST), Vietnam
}

\begin{tabular}{l}
\hline Article Info \\
\hline Article history: \\
Received Oct 8, 2020 \\
Revised Jun 22, 2021 \\
Accepted Jun 25, 2021 \\
\hline Keywords: \\
Car number plate \\
Convolutional neural network \\
Optical character recognition \\
Support vector machine \\
WPOD-NET
\end{tabular}

\begin{abstract}
Nowadays, there are many smart parking lots using plate detection system to control in/out vehicles. However, the disadvantages of systems are a fixed environment and necessity of manual labor and requirement of checkpoints in entrances. To solve the problems, a novel algorithm for wide-angle detecting car number plate using warped planar object detection (WPOD-NET) and a modified support vector machine (SVM) system is proposed. Comparing to other models, the proposal improves not only the range of detection angle but also the accuracy of detecting in shady conditions. The results show that the accuracy of proposal model is up to $95.1 \%$ with 1000 testing images in various scenarios.
\end{abstract}

This is an open access article under the $\underline{C C B Y-S A}$ license.

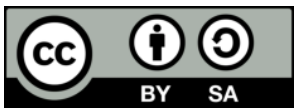

\section{Corresponding Author:}

Phat Nguyen Huu

School of Electronics and Telecommunications

Hanoi University of Science and Technology (HUST)

Vietnam

Email: phat.nguyenhuu@hust.edu.vn

\section{INTRODUCTION}

Nowadays, the automatic transportation detecting system (ATDS) that provides the data of vehicle registration numbers has been developed rapidly. Each car has an identified number to register to law enforcement office before using. Therefore, license plate recognition (LPR) plays an important role in vehicle management system. Deep learning has contributed to enhance the result of computer vision tasks such as object recognition and optical character recognition (OCR). The systems have several applications of traffic and security such as toll fee collection, detecting stolen vehicles, smart house, and parking management. LPR involves capturing the images from a digital camera, pre-processing, and adjusting them for predicting model. Output result is the car plate that appears in the original image. Besides, the system is capable of accommodating to user's requests, for instance manage "smart" parking.

However, most of LPR systems that are set up in Vietnam are designed for recognizing from a fixed view-mostly frontal vehicle in proper environment like indoor scenario or finite externality effects. Therefore, we need to develop the system to solve the problems especially in megacity like Hanoi or Ho Chi Minh where government will apply computer science in managing transportation infrastructure. In the paper, the proposed system using warped planar object detection (WPOD-NET) and a modified support vector machine (SVM) system is performed over various scenarios. As a result, the system is able to be detected Vietnam car plate number in different angles.

The rest of the paper includes five parts and is organized is being as: Section 2 presents several related works. Section 3 presents the proposed model. Section 4 will evaluate the proposal model and analyze the results. In the final section, we give conclusions and future research directions. 


\section{RELATED WORK}

There are many algorithms for detecting car number plate using convolutional neural network (CNN) [1]-[13]. A machine learning model has been proposed based on WPOD-NET [1]. In the model, the authors detect license plate regions of images, regress one affine transformation and resemble them directly. The results are appropriate inputs for the second stage of detection process. CNN model is trained to detect objects (license plates) in different distortions and reformed them to the shape of rectangular that is viewed directly in the front of vehicles. WPOD-NET was built using insights from network single shot MultiBox detector (SSD) [2], you only look once (YOLO) [3], and spatial transformer networks (STN) [4]. SSD and YOLO are in charge of detecting and recognizing multiple objects at the same time. On the other hand, STN is responsible for finding non-rectangular areas although the multi-tasks are out of its possibility. The processing of WPOD-NET is described as:

Firstly, the resized images are transformed into network. It will be extracted the characteristics of smallest region to determine containing the car plate. When feeding the input image into WPOD-NET, the ratio calculating between license plate size and car bounding box is much higher than that of oblique one. Therefore, it may lead to numerical instabilities in output results. Besides, the working principle of network is to use a matrix to transform the imaginary square into a rectangle license plate region. In the next stage, the factor of CNN working have positive results.

In proposal model, CNN-WPOD-NET is used to have the highest accuracy of outputs. In the paper [5], [6] YOLO networks were used to find the region that contains the license plate. The advantage of YOLO network is that the time for detection and accuracy of result are slightly better than WPOD-NET (less than 0.3 second). However, the drawback of their experiment only considers on-plane rotation. As a result, all case of oblique views has been missed without testing. Although YOLO networks work well in finding the whole object in good condition such as in frontal view or not skewing, the results of testing images with different oblique angles are not high.

Duan et al. [7], Smith [8] the authors use Hidden Markov for OCR with the accuracy of the experiment in a specific environment and random ones of $97.82 \%$ and $97.19 \%$, respectively. In the papers, authors consider three fixed angles to capture the images of object (frontal, left and right view) with range of 30 degree. However, the model is not able to detect in case of bad conditions, for example shadow from other objects that affects on the plate and creates uneven color regions. On the other hand, our model has ability to solve the problems of [7], [8] as shown in Table 1.

In [9]-[11], OCR model had been used for experiment. Objects of the paper is Greece car plate that have the similar database of characters and digits with mentioned experiments. Two-layer probabilistic neural network (PNN) gave the result of recognition of $86 \%$ in corresponding scenarios Li et al. [11] without skewed or distorted objects concerned. A failure of detection happened in Roy et al. [9] when objects change to digital image together. The drawback in [9] is similar to that of [8], [12] where the samples are taken image from frontal view. The result is not good when working under poor condition. Moreover, the shape of Greece plate is rectangle that is different from Vietnam car registration plate, and all of the samples are taken from the frontal side. Chen [13], accuracy of system is only $78 \%$ since authors do not apply any filters to reduce the noise before the recognition step.

Berchmans and Kumar [14], authors used a self-organizing (SO) recognition to procedure for their experiment. The procedure consists of three steps, namely character categorization, topological sorting, and recognition. Collecting results was $93.7 \%$ combining for frontal view with a fixed viewpoint and complex scenarios. A salient drawback of paper is the mistake when detecting the same color with vehicles. Besides, the time of characters and digits recognition step is approximately 2 seconds. The same situation also happens with algorithm in [15] where the system takes more than 2 seconds for processing. Xie et al. [5], authors use two Yolo models for detecting and recognizing the characters and digits. However, the samples are on-plane rotation scene. Oblique examples are missed in their experiment.

To solve the problems, we decide to choose the WPOD-NET to recognize the plate based on [5], [8], [9], [11]. Besides, authors [1] also use the same network but their dataset does not contain square plate Vietnam. The proposal model not only detects well in poor condition but also performs adequately in real environment in Vietnam.

Table 1. Comparison of effective algorithms

\begin{tabular}{ccc}
\hline No. & Comparison object & Advance of algorithm \\
\hline 1 & WPOD-NET [9], [11] & Accuracy is 86\% in most challenging scenarios containing oblique license plates \\
2 & SO [14], [15] & Accuracy [14] is 93.7\% while processing time [15] is only 2 seconds \\
3 & Algorithm [13] & 78\% of plate recognition without using noise reduction process \\
4 & Yolo [5] & Using two Yolo models for detecting and recognizing characters and digits \\
\hline
\end{tabular}

Int J Artif Intell, Vol. 10, No. 3, September 2021: 657 - 665 


\section{PROPOSAL SYSTEM}

\subsection{Overview}

Detecting car registration numbers is a small branch of detecting subject. It employs automated methods to verify or recognize the existence of car license plate based on the characteristics of region and shapes. By detecting a rectangle region that contains a group of digits and characters, system is able to find the object of interest (car number plate). System uses pre-trained model and provides information for two main sections, namely law enforcement and commercial applications. Detecting car plate number is used to control the transportation infrastructure and to reduce the damage from congestion to the national economy. Detection system plays an important role in measuring the daily route of vehicles that helps to find the solution for traffic management. In the big cities (Hanoi or Ho Chi Minh), police departments have to maintain the traffic safety and order. Street cameras are set up to supervise moving vehicles and report them. Detecting car registration numbers algorithm consists three major phases, namely bounding box (car plate), detecting the character and digits, and recognizing them as shown in Figure 1. Algorithms containing all listed phased are considered as fully automatic systems and give output results of license plate with the text of digits and characters as shown in Figure 2. In Figure 2(a), we give the result of detecting license plate with front view. Figure 2(b) is the identification result with different angles of license plate. The results show that several number plates are unrecognizable.

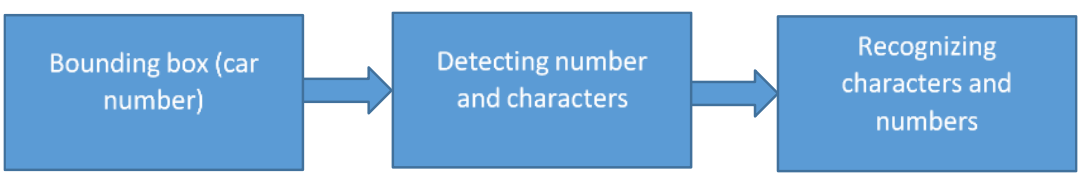

Figure 1. Diagram of car registration numbers detection process

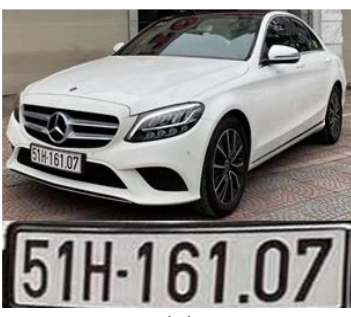

(a)

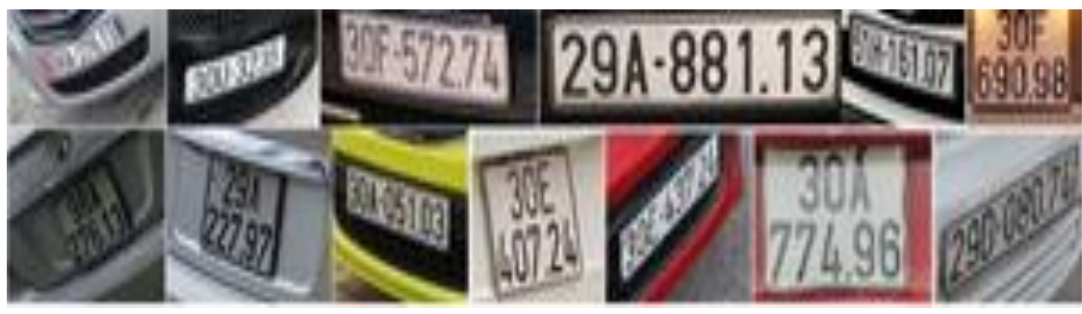

(b)

Figure 2. Result of detecting license plate with digits and characters: (a) an example of detecting car plate, (b) different aspect of a plate

\subsection{Proposal system}

The proposal method includes three steps as shown in Figure 3. In Figure 3(a), we divide into three steps including detecting license plate, extracting characters and digits of plate, and recognizing them. Figure 3(b) shows more detail of performing steps. When we receive an input image, the first module (WPOD-NET) will find the area that has the highest confidence ratio to be the car plate. Since the red, blue, green (RGB) input image of WPOD-NET is setup the dimension from 288 to 608 pixels, images with different sizes are rescaled according to the designed configuration. After reforming, the module skews the detected object to the frontal view corresponding to [1] using $T$ matrix to adjust the angle of characters and digits without losing their features.

In the second step, several obstacles that appears on the plate after changing to grayscale image will appear. They have the approximate values to those of numbers and characters on the plate, and thus it is difficulty to process (problems miss in aforementioned paper). The solution is therefore proposed a practice: using gaussian mask of sigma equal to 10 combining with filters and a threshold. Areas that are not surpass the threshold will be set up to value 1 (white) or 0 (black), respectively. In area inside bounding box (predicting box), OpenCV and other libraries will normalize the object and eliminate noise and obstacles that create problems for the final step. The positive outputs of the second step that is rescaled to the size $30 \times 60$ are taken to the SVM model to predict.

The difference between proposal and [1] is that SVM model is first used as the recognition model, as shown in the first path in Figure 3. The purpose of changing detection model is to improve accuracy and 
time processing of system. Normally, a detection system is designed by neural networks (NN). In contrast, the experiment uses a hybrid system combining two heterogeneous model for application of "smart" parking for significant reasons:

- Small, required data source for SVM compared to NN.

- Non-trivial parameter optimization (SVM just requires 2-3 parameters).

- SVM is more interpretable than NNs.

- Commercial product needs to low price without changing the accuracy of system since SVM is the best choice.

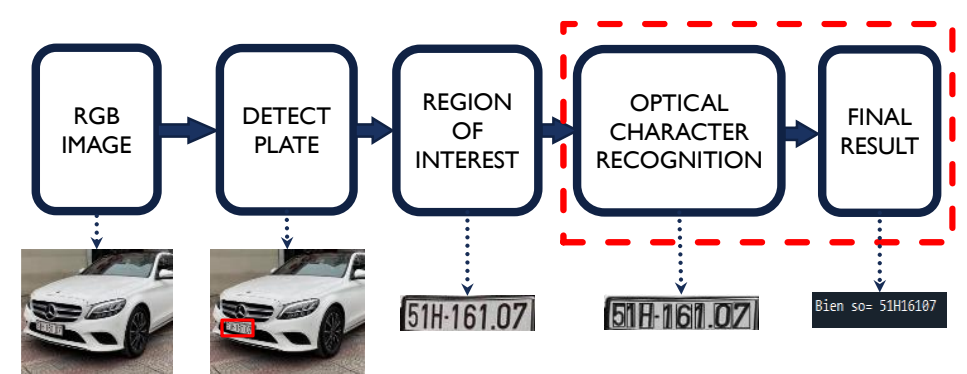

(a)

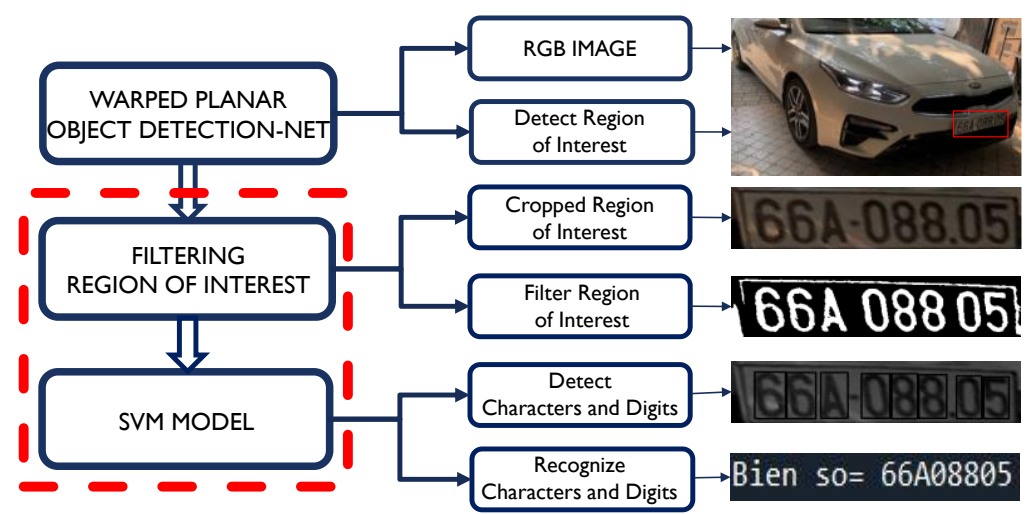

(b)

Figure 3. Three processing steps of proposal system: (a) detecting license plate, extracting characters, digits of plate, and recognizing steps, (b) detailed steps to implement blocks

\subsubsection{Recognizing car plate}

Detecting license plate, being the first phase has important role of following steps and directly affects to the output result. Most automated system using to detect car registration numbers performs for a fixed environment. Besides, the diversity of shape and place where car plate set up creates challenges, as shown in Figures 4 and 5 for the system to have the optimal output. Manual activities proceed to collect the information that requires from the beginning instead of totally replacing by the computer.

In the paper, we choose WPOD-NET model to recognize the plate. WPOD-NET consists of 21 convolutional layers and 14 of them are inside residual blocks. The size of all internal filter is $3 \times 3$ and ReLU activations are the algorithms using in the network excepting the detection block 4 max pooling layers (size $2 \mathrm{x} 2$ ) with stride 2 that decreases the input image by a factor of 16. In the final box, there are two parallel layers to submit for two cases: one infers the probability that is operated by SOFTMAX function, one uses linear function. More details can be seen in [1].

\subsubsection{Detecting characters and digits}

In the second phase, the automated system will use an algorithm to detect the characters and digits inside the object that is found in the previous phase. By using Python and library such as TensorFlow, open source computer vision library (OpenCV), numerical python (NumPy) or PIL to process the images before starting to detect, the region has the highest probabilities to be a digit or character. The results are shown in Figure 6. Figure 6(a) is an example of detecting characters and digits of license plate by using TensorFlow. 
Figure 6(b) is the input images with different viewing angles. They will be used for identification and classification in the next steps.

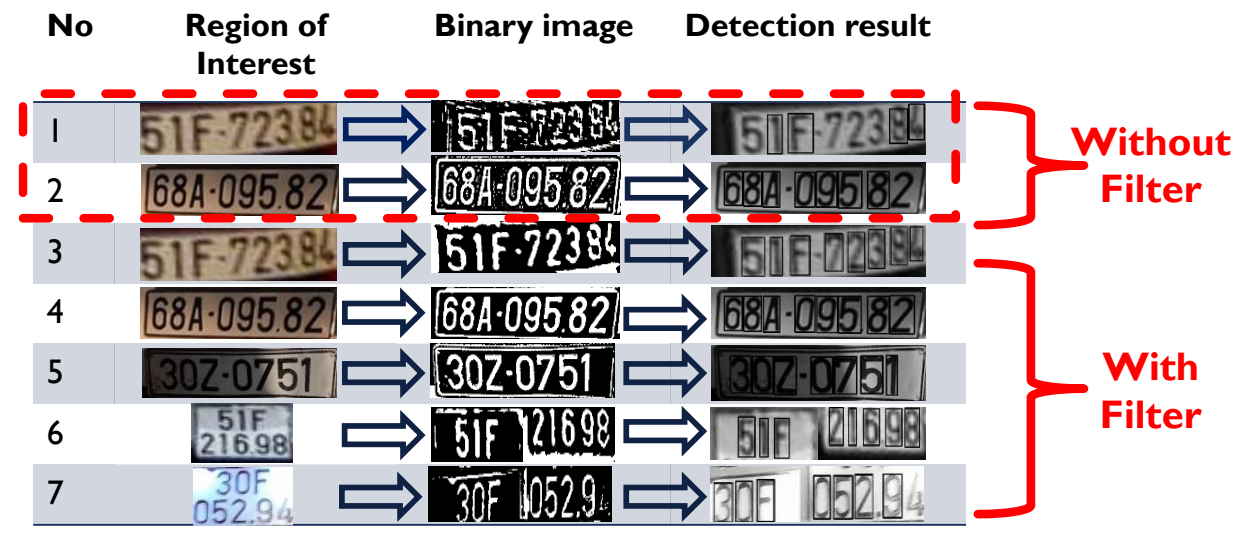

Figure 4. Result of region of interest in good and bad cases

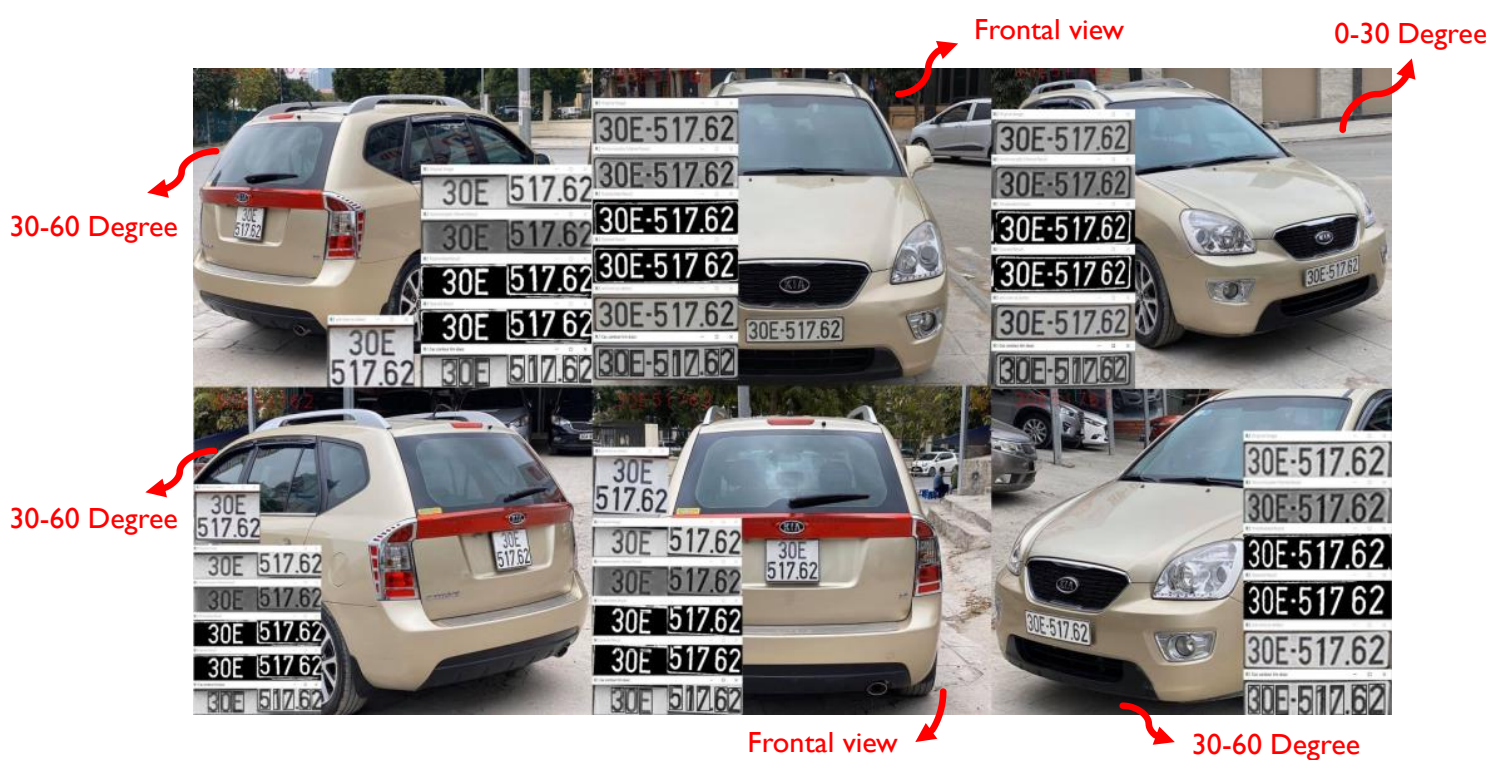

Figure 5. Detecting result of different angles

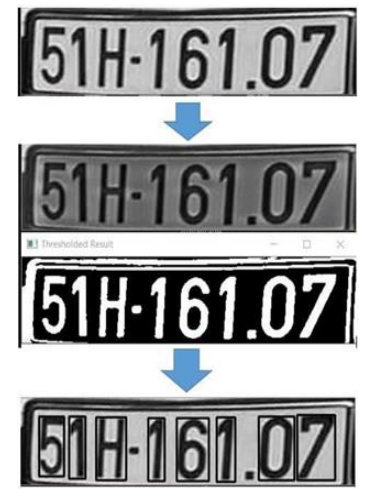

(a)

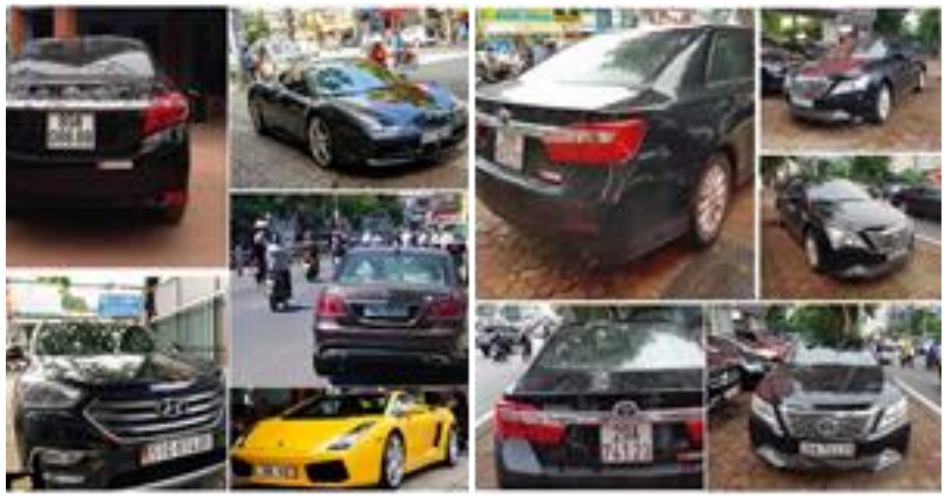

(b)

Figure 6. Result of detecting license plate in the second phase, (a) process of detecting characters and digits, (b) different cases of test sample 


\subsubsection{Recognizing characters and digits}

There are many methods to recognize figures and the most well-known libraries have been used SVM [16], [17] and tesseract optical character recognition (OCR) [18], [19].

- Tesseract OCR engine: This is a top engine in the world. It has been distributed with open-source Apache 2.0 that supports recognition characters in images and extracts them into raw material, html, pdf, tsv. Its function can be used through API. Tesseract OCR is an open-source project starting by Hewlett-Packard. In 2018, the latest stable version 4.0.0 is based on long short-term memory (LSTM). LSTM is a famous form of recurrent neural network (RNN) and used to solve the text of arbitrary length. Furthermore, it supports many image formats and is gradually added a large number of languages.

- SVM: Model analyzes data using for classification and regression. SVMs are considered as the highest classification accuracy as a binary classifier [20]-[22]. It is the learning technique that is considered an effective method for general purpose because of its high performance without adding other knowledges. At the beginning state, SVM finds the hyperplanes (decision boundaries) that classify the data. It performs to separate the largest possible fraction of points of the same class on one side while optimizing the distance from either class to hyperplane. This hyperplane is called optimal separating hyperplane $(\mathrm{OSH})$ that minimizes the risk of misclassifying not only the examples in the training dataset but also the unseen example. There are several advantages of SVM model: i) It is a very good algorithms for the unknown database, ii) It is appropriate for specific working background similar to text classification, iii) It has great possibility in scaling to high dimensional data.

Due to the fact that each region in the world uses a different font for characters and digits on the plate, we used a different dataset for Vietnam car plates. The recognition module is a support vector machine model. The primary reason for choosing an SVM model is that it only requires a smaller data source compared to a neural network. Besides that, an SVM model only requires three parameters to setup. SVM is popular in text classification tasks, where consider the norm is high-dimensional spaces. In this paper, a type of SVM is used for OCR module-C-SVM. 36 groups of characters and digits in binary format are separated by hyperplanes with penalty multiplier $\mathrm{C}$ equals 1 for outlier.

There is an obstacle that affects to detecting results for poor image quality. When the features are extracted, the discrimination functions between each pair are learned by SVMs. Therefore, a binary tree structure to recognize the testing samples is proposed to construct in the paper. For detecting characters and digits, multi-class SVM is used to assign labels to instances. The approach to the problem creates a difficulty of multiple binary classification. The common method is to distinguish one object from all others. It is performed based on [17] that have the classifier with highest output function.

\section{SIMULATION AND RESULTS}

\subsection{Setup}

WPOD-net combining with SVMs algorithm is used to detect the car registration numbers in the paper. The testing data consists of 1000 images of vehicles for reality scenarios. In our experiment, each of the object has three angles of license plate including on left, right, and in front of. There are four cases of bad conditions, namely in the evening, in the shadow of tree, lack of brightness, and faded numbers of plates. Several of them are slightly blurred or distorted. Distance from camera to plate is variance to consider and it is manifested through plate and original image. Most of them are in good condition with clear view. We divided two main groups of license plate, namely one for random object and one for a group with different angles of each car. Algorithm is performed by LG Gram Intel ${ }^{\circledR}$ Core $^{\mathrm{TM}}$ i5-7200 CPU @ 2.50GHz $2.71 \mathrm{GHz}$ with 7.86 GB RAM, 64-bit Windows 10. The ratio between training and testing dataset is 4:1.

A few cases show that the system has a mistake in determining whether square plate and rectangle plates. As a result, the output results are not correct. Due to different angles of the objects, there are several mistaken shape classifications.

\subsection{Results}

There are several salient instances as shown in Table 2. Those are images of vehicle are captured in challenging scenarios by ourselves. We also use the filter to check the quality of images when apply algorithm. As a result, the algorithm failed to identify the characters and digits on plate without the filter as shown in Figure 4. On the other hand, several cases are identified fully the characters and digits with filter.

In Tables 2 and 3, there are several cases that contains plate with complex scenarios. The first three cases are applied Gaussian mask to improve the output results for detection step where all characters and digits are recognized. No. 4 and 5 do not apply the noise filter, and thus result in recognition step is not good. No. 6 is the case that image is affected by streetlight. As a result, no. 4 is missed in predicting step. No. 7 is 
an example of our system executing in shady condition with uneven brightness. By using filter, the output is $100 \%$ correct recognition.

Our goal of experiment is to recognize correct the whole string of characters and numbers of the plate. Final result contains 951 correct images with full sequence numbers and characters of objects. On the other hand, 49 incorrect results give outputs with mistaken numbers and characters or missing object due to conditional environment. As shown in Tables 2 and 3, most of incorrect results happen for 42 cases since recognition system has limitation in detecting characters and digits in plates that is transformed from oblique views. Besides, seven objects taking images from frontal views have incorrect outputs because of common errors such as mistaking in recognition of B and 8 or the brightness of environment. Figures 5 and 7 illustrate intuitive scenarios of smart parking. Processing time to detect the plate is from 0.7 to 1.2 seconds (depending on the change of environment and quality of images). However, the result of proposal is not good in several cases based on the weather condition, the blurring problem, or shadow of object covers. We will therefore improve the system to solve the problems and combine others advantaged networks [23]-[26] to improve the accuracy.

Table 2. Result of proposal algorithm

\begin{tabular}{|c|c|c|c|c|c|c|c|c|c|c|c|}
\hline \multirow[t]{3}{*}{ Type of object } & \multirow{3}{*}{$\begin{array}{c}\text { Number } \\
\text { of } \\
\text { objects }\end{array}$} & \multirow{2}{*}{\multicolumn{4}{|c|}{$\begin{array}{c}\text { Different scenarios } \\
\text { Distance }(\mathrm{m}) \text { Range of angle }\end{array}$}} & \multicolumn{4}{|c|}{ Number of correct detections } & \multirow{3}{*}{$\begin{array}{l}\text { Ratio of correct } \\
\text { detection }(\%)\end{array}$} & \multirow{3}{*}{ Quality of object } \\
\hline & & & & & & \multicolumn{2}{|c|}{ Distance $(\mathrm{m})$} & \multicolumn{2}{|c|}{ Range of angle } & & \\
\hline & & $0-1$ & $1-2$ & $0-30$ & $30-60$ & $0-1$ & $1-2$ & $0-30$ & $30-60$ & & \\
\hline 1. Frontal view & 500 & 290 & 210 & $\mathrm{x}$ & $\mathrm{x}$ & 288 & 205 & $\mathrm{x}$ & $x$ & 98.6 & All objects are in \\
\hline 2. Oblique views & 500 & 240 & 260 & 300 & 200 & 210 & 248 & 284 & 174 & 91.2 & yinal shape, \\
\hline 2.1. Left View & 200 & 100 & 100 & 125 & 75 & 79 & 96 & 113 & 62 & 86.5 & without physical \\
\hline 2.2. Right View & 300 & 140 & 160 & 175 & 125 & 131 & 152 & 171 & 112 & 94.3 & transformation \\
\hline TOTAL & 1000 & & & 000 & & & & 51 & & 95.1 & \\
\hline
\end{tabular}

Table 3. Results of complex cases

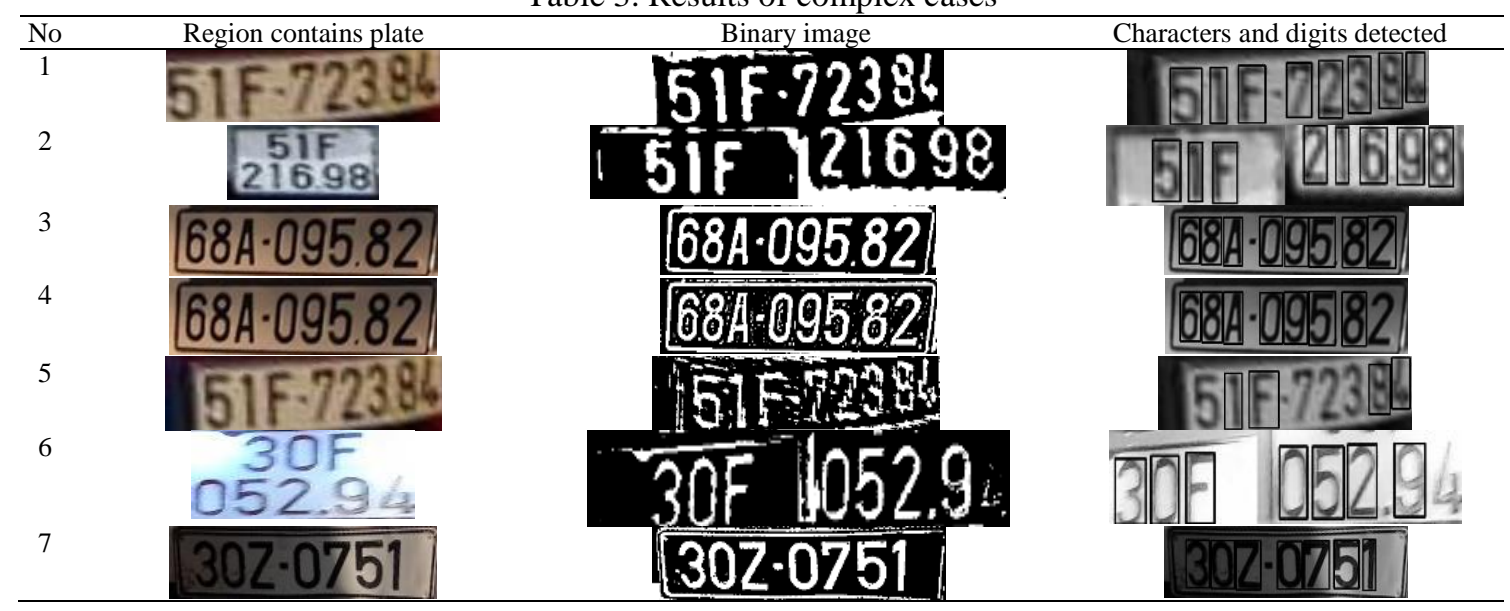

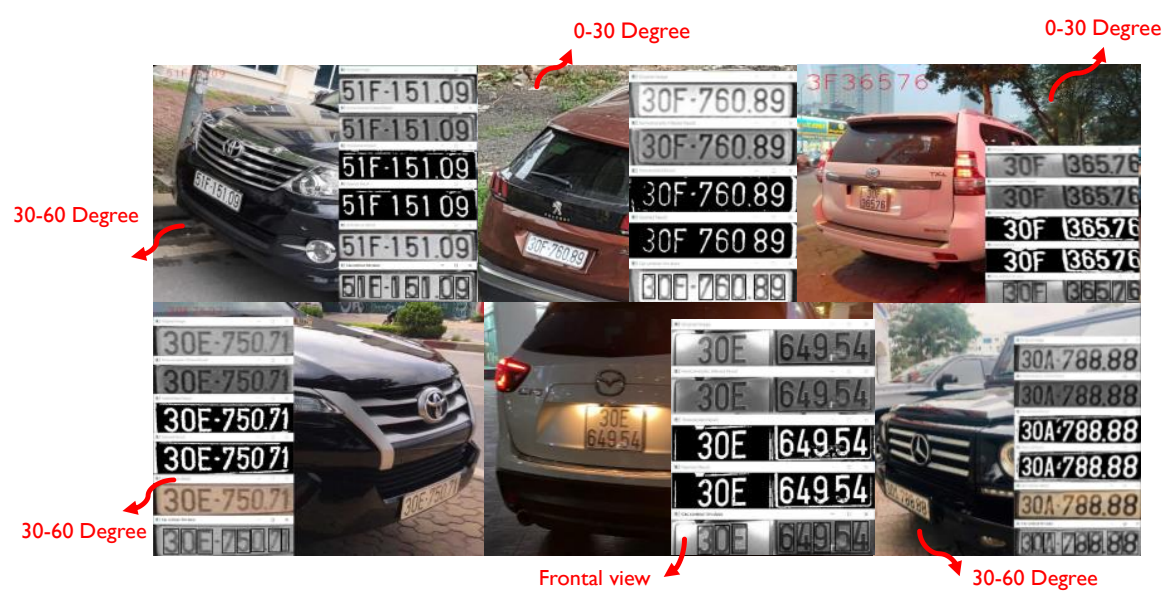

Figure 7. The samples contain different distance and angle 


\section{CONCLUSION}

This paper proposes the car numbers detection algorithm for parking systems. The system needs to improve the step of increasing standard of object detected images. In the real applications, environment plays an important role of detection. The factors include: the camera has low quality; the weather condition is bad; or the blurring problem or shadow of object covers the plate. All problems cause to decrease the resolution of plate detection system. The shadow creates different brightness in the plate that makes the system to be unable to normalize the images for further steps. Therefore, we will create an adaptive wavelet filter optimizing the pre-process module and combine with other networks to improve the accuracy for the proposal system.

\section{ACKNOWLEDGEMENTS}

This research is carried out in the framework of the project funded by the ministry of education and training (MOET), Vietnam under the grant B2020-BKA-06. The authors would like to thank the MOET for their financial support.

\section{REFERENCES}

[1] S. M. Silva and C. R. Jung, "License plate detection and recognition in unconstrained scenarios," Proceedings of the European conference on computer vision (ECCV), vol. 11216, 2018, pp. 580-596.

[2] W. Liu et al., "SSD: Single shot multibox detector," in Proceedings of the European Conference on Computer Vision (ECCV), Springer, Cham, vol. 9905, 2016, pp. 1-15, doi: 10.1007/978-3-319-46448-0_2.

[3] J. Redmon and A. Farhadi, "Yolov3: An incremental improvement," arXiv preprint arXiv:1804.02767, 2018. [Online]. Available: http://arxiv.org/ abs/1804.02767.

[4] M. Jaderberg, K. Simonyan, A. Zisserman, and K. Kavukcuoglu, "Spatial transformer networks," arXiv preprint arXiv:1506.02025, 2015.

[5] L. Xie, T. Ahmad, L. Jin, Y. Liu, and S. Zhang, "A New CNN-Based Method for Multi-Directional Car License Plate Detection," in IEEE Transactions on Intelligent Transportation System, vol. 19, no. 2, pp. 507-517, 2018, doi: 10.1109/TITS.2017.2784093.

[6] S. Montazzolli and C. Jung, "Real-Time Brazilian License Plate Detection and Recognition Using Deep Convolutional Neural Networks," 2017 30th SIBGRAPI Conference on Graphics, Patterns and Images (SIBGRAPI), 2017, pp. 55-62, doi: 10.1109/SIBGRAPI.2017.14.

[7] T. Duan, D. Tran, P. Tran, and N. Hoang, "Building an automatic vehicle license-plate recognition system," in International Conference in Computer Science RIVF05, Can Tho, Vietnam, Feb. 2005, pp. 59-63.

[8] R. Smith, "An Overview of the Tesseract OCR Engine," Ninth International Conference on Document Analysis and Recognition (ICDAR 2007), 2007, pp. 629-633, doi: 10.1109/ICDAR.2007.4376991.

[9] K. Roy et al., "An analytical approach for enhancing the automatic detection and recognition of skewed bangla license plates," in International Conference on Bangla Speech and Language Processing (ICBSLP), 2019, pp. 1-4.

[10] C. N. E. Anagnostopoulos, I. E. Anagnostopoulos, V. Loumos, and E. Kayafas, "A License Plate-Recognition Algorithm for Intelligent Transportation System Applications," in IEEE Transactions on Intelligent Transportation Systems, vol. 7, no. 3, pp. 377-392, Sept. 2006, doi: 10.1109/TITS.2006.880641.

[11] H. Li, P. Wang, and C. Shen, "Toward End-to-End Car License Plate Detection and Recognition with Deep Neural Networks," in IEEE Transactions on Intelligent Transportation Systems, vol. 20, no. 3, pp. 1126-1136, 2019, doi: 10.1109/TITS.2018.2847291.

[12] Shyang-Lih Chang, Li-Shien Chen, Yun-Chung Chung, and Sei-Wan Chen, "Automatic license plate recognition," in IEEE Transactions on Intelligent Transportation Systems, vol. 5, no. 1, pp. 42-53, March 2004, doi: 10.1109/TITS.2004.825086.

[13] R.-C. Chen, "Automatic License Plate Recognition via sliding-window darknet-YOLO deep learning," Image and Vision Computing Journal, vol. 87, pp. 47-56, 2019, doi: 10.1016/j.imavis.2019.04.007.

[14] D. Berchmans and S. S. Kumar, "Optical character recognition: An overview and an insight," 2014 International Conference on Control, Instrumentation, Communication and Computational Technologies (ICCICCT), 2014, pp. 1361-1365, doi: 10.1109/ICCICCT.2014.6993174.

[15] H. Li, P. Wang, M. You, and C. Shen, "Reading car license plates using deep neural networks," Image and Vision Computing Journal, vol. 72, pp. 14-23, 2018, doi: 10.1016/j.imavis.2018.02.002.

[16] C. Nello and S-T. John, "An introduction to support vector machines and other kernel-based learning methods. Repr," Introduction to Support Vector Machines and other Kernel-Based Learning Methods, vol. 22, Jan. 2001, doi: $10.1017 /$ CBO9780511801389.

[17] T. C. Mota and A. C. G. Thome, "One-Against-All-based multiclass SVM strategies applied to vehicle plate character recognition," 2009 International Joint Conference on Neural Networks, 2009, pp. 2153-2159, doi: 10.1109/IJCNN.2009.5178902.

[18] L. Zheng, X. He, Q. Wu, and T. Hintz, "Number plate recognition without segmentation," Proceedings of Image and Vision Computing New Zealand, 2007, pp. 164-168. 
[19] M. G. Marne, P. R. Futane, S. B. Kolekar, A. D. Lakhadive and S. K. Marathe, "Identification of Optimal Optical Character Recognition (OCR) Engine for Proposed System," 2018 Fourth International Conference on Computing Communication Control and Automation (ICCUBEA), 2018, pp. 1-4, doi: 10.1109/ICCUBEA.2018.8697487.

[20] M. A. Hearst, S. T. Dumais, E. Osuna, J. Platt, and B. Scholkopf, "Support vector machines," in IEEE Intelligent Systems and their Applications, vol. 13, no. 4, pp. 18-28, July-Aug. 1998, doi: 10.1109/5254.708428.

[21] K. I. Kim, K. Jung, S. H. Park, and H. J. Kim, "Support vector machines for texture classification," in IEEE Transactions on Pattern Analysis and Machine Intelligence, vol. 24, no. 11, pp. 1542-1550, Nov. 2002, doi: 10.1109/TPAMI.2002.1046177.

[22] G. Wang, "A Survey on Training Algorithms for Support Vector Machine Classifiers," 2008 Fourth International Conference on Networked Computing and Advanced Information Management, 2008, pp. 123-128, doi: 10.1109/NCM.2008.103.

[23] P. N. Huu, T. Tran Van, and N. G. Thi, "Proposing distortion compensation algorithm for determining distance using two cameras," 2019 6th NAFOSTED Conference on Information and Computer Science (NICS), 2019, pp. 172-177, doi: 10.1109/NICS48868.2019.9023875.

[24] T. N. Duong, V. D. Than, T. H. Tran, Q. H. Dang, D. M. Nguyen, and H. M. Pham, "An effective similarity measure for neighborhood-based collaborative filtering," in 2018 5th NAFOSTED Conference on Information and Computer Science (NICS), 2018, pp. 250-254, doi: 10.1109/NICS.2018.8606859.

[25] S. Ghosh, A. Dasgupta and A. Swetapadma, "A Study on Support Vector Machine based Linear and Non-Linear Pattern Classification," 2019 International Conference on Intelligent Sustainable Systems (ICISS), 2019, pp. 24-28, doi: 10.1109/ISS1.2019.8908018.

[26] C. T. Phan, D. D. Pham, H. V. Tran, and P. N. Huu, "Applying the IoT platform and green wave theory to control intelligent traffic lights system for urban areas in Vietnam," KSII Transactions on Internet and Information Systems, vol. 13, no. 1, pp. 34-51, Jan. 2019, doi: 10.3837/tiis.2019.01.003.

\section{BIOGRAPHIES OF AUTHORS}

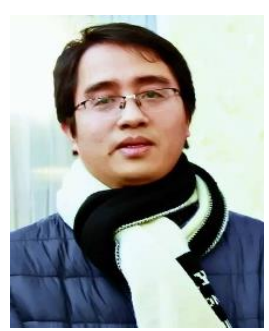

Phat Nguyen Huu received his B.E. (2003), M.S. (2005) degrees in Electronics and Telecommunications at Hanoi University of Science and Technology (HUST), Vietnam, and Ph.D. degree (2012) in Computer Science at Shibaura Institute of Technology, Japan. Currently, he lecturer at School of Electronics and Telecommunications, HUST Vietnam. His research interests include digital image and video processing, wireless networks, ad hoc and sensor network, and intelligent traffic system (ITS) and internet of things (IoT). He received the best conference paper award in SoftCOM (2011), best student grant award in APNOMS (2011), hisayoshi yanai honorary award by Shibaura Institute of Technology, Japan in 2012.

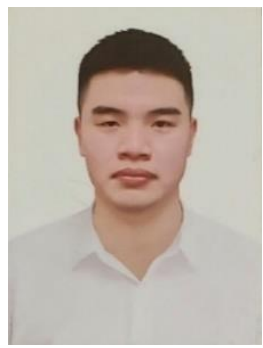

Cuong Vu Quoc is student of Electronic and Telecommunications at Hanoi University of Science and Technology (HUST), Vietnam. Currently, he is working in Future Network lab. at HUST. His main duty is developing smart products which relates to digital image, video processing, machine learning and internet of things (IoT). 